PoS $\quad \begin{aligned} & \text { PROCEEDINGS } \\ & \text { OF SCIENCE }\end{aligned}$

\title{
The 9th International Workshop on Chiral Dynamics 2018
}

Mohammad W. Ahmed* North Carolina Central University and TUNL

E-mail: ahmedetunl.duke.edu

Haiyan Gao Duke University and TUNL

E-mail: gao@tunl.duke.edu

Calvin R. Howell Duke University and TUNL

E-mail: howell@tunl.duke.edu

Dean Lee Michigan State University

E-mail: leedefrib.msu.edu

The 9th International workshop on Chiral Dynamics

17-21 September 2018

Durham, NC, USA

${ }^{*}$ Speaker. 


\section{Preface}

The 9th International Workshop on Chiral Dynamics 2018 brought the series back to the Triangle Universities Nuclear Laboratory (TUNL) in Durham, NC since its 5th gathering in 2006. The field of Chiral Dynamics has matured over the 25-year span of the series which started in 1994 at MIT, followed by Mainz (1997), Jefferson Lab (2000 and 2012), Bonn (2003), Duke (2006, 2018), Bern (2009), and Pisa (2015). The workshop has traditionally drawn equal participation from theorists and experimentalists to showcase the most recent advances in the field, and to discuss future directions. The 9th workshop maintained the organization of the scientific program into topical groups of: Hadron Structure, Goldstone-Boson Dynamics, and Few-Body Dynamics. As observed elsewhere in the field, the workshop illuminated the increased effort in the lowenergy QCD community of using lattice simulation techniques applied across the sub-fields and the increased demand for solutions from lattice calculations. Nearly 120 participants attended the workshop, which delivered twenty plenary talks, and over ninety parallel presentations. Research highlights presented in this workshop include results from muon g-2 measurements, calculations of the properties of light nuclei in lattice QCD, studies of the spin structure of the nucleon, pion life-time measurements, determinations of the electromagnetic and spin polarizabilities of nucleons via Compton scattering, applications of chiral dynamics in the electroweak sector, and various topics treated in the framework of lattice chiral effective field theory. The program also included a prelude to the physics opportunities at a future US based electron-ion collider. In addition, the workshop welcomed an increased participation from colleagues in Asia. To further facilitate this development, and the increased interest in the field from the Asian region, for the first time in the history of this workshop series, the organizers have selected the next workshop to be held in Beijing, China. These proceedings present topics convened in this 9th workshop and provide a comprehensive review of the field, highlighting recent accomplishments and discussing challenges that will motivate research in the coming years. We look forward to gathering and discussing exciting new developments in the field in Beijing in 2021.

Chairs,

Mohammad W. Ahmed

Haiyan Gao

Calvin R. Howell, and

Dean Lee

September 17-21, 2018, Durham, NC 


\section{International Advisory Committee}

Balasubramanian Ananthanarayan Mario Antonelli

Sinya Aoki

Silas Beane

Paulo Bedaque

Veronique Bernard

Aron Bernstein

Johan Bijnens

Michael Birse

Jian-Ping Chen

Vincenzo Cirigliano

Gilberto Colangelo

Evgeny Epelbaum

Ruben Flores-Mendieta

Simona Giovannella

Josè Goity

Harald Griesshammer

Feng-Kun Guo

Barry Holstein

Karl Jansen

Marc Knecht

Heinrich Leutwyler

Laura Marcucci

Judith McGovern

Ulf-G Meissner

Harald Merkel

Jose Pelaez

Daniel Phillips

Christopher Sachrajda

Toru Sato

Martin Savage

Norberto Scoccola

Stephen Sharpe

Bira Van Kolck

Marc Vanderhaegen

Michele Viviani

Hartmut Wittig
Indian Institute of Science

National Institute for Nuclear Physics, INFN

University of Tsukuba

Institute for Nuclear Theory, Univ. of Washington

University of Maryland

Institut de Physique Nucleaire

Massachusetts Institute of Technology

University of Lund

University of Manchester

Jefferson Laboratory (JLab)

Los Alamos National Laboratory

University of Bern

University of Bochum

University of San Luis Potosi

National Institute for Nuclear Physics, INFN

Hampton University \& JLab

George Washington University

Institute of Theoretical Physics, CAS

University of Massachusetts

Deutsches Elektronen-Synchrotron (DESY)

Centre National de la Recherche Scientifique

University of Bern

University of Pisa

University Manchester

University of Bonn \& FZ-Julich

Institut fur Kernphysik

University of Madrid

Ohio University

University of Southampton

Osaka University

Institute for Nuclear Theory, Univ. of Washington

Comision Nacional de Energia Atomica

University of Washington

University Arizona

University of Mainz

University of Pisa

University of Mainz
Bangalore, India

Frascati, Italy

Tsukuba, Japan

Seattle, USA

Maryland, USA

Orsay, France

Boston, USA

Lund, Sweden

Manchester, UK

Newport News, USA

USA

Bern, Switzerland

Bochum, Germany

Mexico

Frascati, Italy

Newport News, USA

Washington, DC, USA

Beijing, China

Amherst, USA

Zeuthen, Germany

Marseille, France

Bern, Switzerland

Italy

Manchester, UK

Bonn, Germany

Mainz, Germany

Madrid, Spain

Athens, USA

UK

Japan

Seattle, USA

Argentina

Seattle, USA

Tucson, USA

Mainz, Germany

Italy

Germany 


\section{Working Group Conveners}

$\begin{array}{ll}\text { Goldstone-Boson Dynamics } & \text { Jacobo de Elvira } \\ & \text { Jose Luis Goity } \\ & \text { Liping Gan } \\ & \text { Simona Giovannella } \\ & \text { Carsten Urbach } \\ & \text { Jian-Ping Chen } \\ & \text { William Detmold } \\ & \text { Harald Griesshammer } \\ \text { Hadron Structure } & \text { Hermann Krebs } \\ & \text { Rory Miskimen } \\ & \text { Jose Manuel Alarcón Soriano } \\ & \text { Evgeny Epelbaum } \\ & \text { Douglas Higinbotham } \\ \text { Few-Body Dynamics } & \text { Daniel Phillips } \\ & \text { Werner Tornow }\end{array}$

University of Bern

Jefferson Laboratory

University of North Carolina - Wilmington

National Institute for Nuclear Physics, INFN

University of Bonn

Jefferson Laboratory

Massachusetts Institute of Technology

George Washington University

University of Bochum

University of Massachusetts

Jefferson Laboratory

University of Bochum

Jefferson Laboratory

Ohio University

TUNL \& Duke University 